\title{
Global exponential stability result for complex valued hysteretic neuron model
}

\section{G. Padmavathi}

Department of Mathematics, JNTUH College of Engineering, Kukatpally, Hyderabad-72, Andhra Pradesh, India and CR Rao AIMSCS, University of Hyderabad Campus, P.O. Central University, Hyderabad-500 046, Andhra Pradesh, India E-mail: padmagvathi@yahoo.co.in

\section{P.V. Siva Kumar*}

CSE Department, VNR VJIET, Bachupally, Nizampet (S.O), Hyderabad-090, Andhra Pradesh, India E-mail: sivakumar_pv@vnrvjiet.in *Corresponding author

\section{Shahnaz Bathul}

Department of Mathematics, JNTUH College of Engineering, Kukatpally, Hyderabad-72, Andhra Pradesh, India E-mail: shahnazbathul@yahoo.com

\begin{abstract}
A mathematical model of artificial neural networks with hysteresis is formulated using neutral delay differential equations. Hysteresis modifies the systems such that they cannot produce unique output for any given input, rather output is produced based on the past history of the system. Motivated by the applications of complex valued neural networks in artificial neural networks, we studied the global dynamics of complex valued neural network with hysteresis. The result extends and improves the earlier publications due to the fact that it removes some restrictions on the neural delay. In this paper continuous hysteresis neuron model has been used to arrive at the sufficient condition for global exponential stability of a unique equilibrium. The hypothetical insight has been successfully applied and verified using relevant numerical examples.
\end{abstract}

Keywords: discretisation; hysteretic neural network; activation dynamics.

Reference to this paper should be made as follows: Padmavathi, G., Kumar, P.V.S. and Bathul, S. (2013) 'Global exponential stability result for complex valued hysteretic neuron model', Int. J. Machine Intelligence and Sensory Signal Processing, Vol. 1, No. 1, pp.23-45. 
Biographical notes: G. Padmavathi has received her gold medal in MSc (Maths) from Acharya Nagarjuna University, Guntur, Andhra Pradesh, India in 2000 and received MPhil from Madurai kamaraj University, Tamil Nadu in 2003. Currently, she is pursuing her PhD at JNTUH University, Hyderabad. Her main research interest includes differential equations, modeling and analysis. She also selected for Women Scientist under WOS-A, DST with the File no. SR/WOS-A/MS-05/2011.

P.V. Siva Kumar received his Master of Computer Applications from JNTU Hyderabad in 1999 and MTech in Computer Science and Engineering from Vinayaka Missions University in 2007. Currently, he is pursuing his $\mathrm{PhD}$ in Computer Science and Engineering from Acharya Nagarjuna University, Guntur, Andhra Pradesh, India. He is presently working as an Associate Professor in CSE Department at VNR VJIET Hyderabad. His current research interests computer networks, network security and information security. He is a life member of CSI and ISTE.

Shahnaz Bathul is a Professor in the Department of Mathematics at JNTUH College of Engineering, Kukatpally, Hyderabad, India. She received her PhD from IIT Karagpur in 1984. She has more than two decades of teaching and research experience. She is a member of Indian Statistical Association and Mathematical Association of America. Her research interests include fluid dynamics, dynamical systems numerical analysis, operations research, probability and statistics.

\section{Introduction}

Hysteresis is normally observed in several engineering systems such as control systems, electronic circuits, and piezoelectric actuator, etc. This phenomena also observed in animals such as frogs (Didday, 1976) and crayfish (Hale, 1977). The hysteretic neural network model is envisaged to be efficient and robust for various applications such as medical image processing, military data processing, etc. Hysteretic feedback control phenomena also manage glucose vs. lactose utilisation preference in Escherichia coli and ensure unidirectional cell-cycle progression in eukaryotes. Activation models of neural network with past history produce hysteresis effects and study of their dynamics has been the subject of recent investigations. Hoffman (1986) has observed that there are several possible biochemical schemes that lead to hysteresis. Continuous time dependent models of hysteretic neural networks have also been identified by several researchers (Hoffman, 1986; Hoffman and Benson, 1986; Segundo and Martinez, 1985; Macki et al. 1993; Gopalsamy and Liu, 2007; Krasnosel'skii and Pokrovskii, 1989; Feckan, 1999), which can be mathematically described by the following state equations.

$$
x_{i}^{\prime}(t)=-a_{i} x_{i}(t)+\sum_{k=1}^{m} b_{i k} f_{k}\left(x_{k}(t)+c_{k} x_{k}(t-\tau)+p_{k} \dot{x}_{k}(t-\sigma)+q_{k}\right), t>0 .
$$

here ' $=\frac{d}{d t}, x(t)=\left[x_{1}(t), \ldots \ldots, x_{m}(t)\right]^{T} \in C^{m}$, and the activation function $f(x(t))=\left[f\left(x_{1}(t)\right)\right.$, $\left.\ldots \ldots, f\left(x_{m}(t)\right)\right]^{T} \in C^{m}, a_{i}, c_{i}, p_{i}, q_{i} \in R^{m}, i=1,2, \ldots \ldots m$ and the connection weights 
$\tilde{M}=\left[b_{i k}\right]_{m \times m}$ assumed to be complex valued. In which passive decays $a=\operatorname{diag}\left\{a_{i}>0\right\}$ (a positive diagonal matrix) $a_{i}$ denotes the range of continuous variable, $c=\operatorname{diag}\left\{c_{1}, c_{2}, \ldots \ldots c_{m}\right\}, c_{i}$ denotes the measure of the inhibitory influence of the past information, $p=\operatorname{diag}\left\{p_{1}, p_{2}, \ldots \ldots p_{m}\right\}, p_{i}$ denotes the measure of rate of change inhibitory influence of the past history due to deactivation. The functions $f_{k}, k=1,2, \ldots n$ are complex valued continuous and are defined on $(-\infty, \infty), q_{j}$ denotes the external input vector and the delays $\sigma>0, \tau>0$ are positive. $x_{k}(t)$ in the argument of $f_{k}$ function in (1) denotes a local positive feedback, in neural network literature these are known as self excitations while in the biological literature which are known as reverberations.

Complex valued neural networks have been found to be useful in extending the scope of application in optoelectronics, imaging, remote sensing, quantum neural devices and systems and artificial neural information processing. Applications of these networks can be found in Hirose (2003). The difficulties that arise out of changing the real variables to complex variables have been well explained by Georgion and Koutsougeras (1992) (Sreeharirao and Ramamurthy, 2008). Complex-valued neural networks deal with not only complex-valued signals but also have the following excellent properties (Nitta, 1997).

1 the average convergence speed is two or three times faster than that of real-valued neural networks

2 the number of required hidden parameters is approximately half that of real-valued neural networks.

It is essential to obtain discretisation of the continuous-time neural network for real time applications and implementation of computer simulations. Due to this, it is also necessary to study the stability of discrete time neural networks from both theoretical and practical point of view. By a proper approximation (Gopalsamy and Mohamad, 2003), obtained the following discretisation scheme of system (1) and have shown that this discrete-time analogue can preserve the convergence dynamics of the corresponding continuous-time version (1).

Consider (1)

$$
\frac{d x_{i}(t)}{d t}=-a_{i} x_{i}(t)+\sum_{k=1}^{m} b_{i k} f_{k}\left(y_{k}(t)\right),
$$

where $y_{i}(t)=x_{i}(t)+c_{i} x_{i}(t-\tau)+p_{i} \dot{x}_{i}(t-\sigma)+q_{i}, i=1,2, \ldots \ldots m$.

Which implies that

$$
\begin{aligned}
y_{i}(t)= & x_{i}\left(\left[\frac{t}{h}\right] h\right)+c_{i} x_{i}\left(\left[\frac{t}{h}\right] h-\left[\frac{\tau}{h}\right] h\right) \\
& +p_{i}\left[x_{i}\left(\left[\frac{t}{h}\right] h-\left[\frac{\sigma-1}{h}\right] h\right)-x_{i}\left(\left[\frac{t}{h}\right] h-\left[\frac{\sigma}{h}\right] h\right)\right]+q_{i} .
\end{aligned}
$$


Since

$$
\begin{aligned}
\dot{x}_{i}(t-\sigma) & =\lim _{h \rightarrow 0} \frac{x_{i}(t-\sigma+h)-x_{i}(t-\sigma)}{h} \\
& \approx x_{i}(t-\sigma+1)-x_{i}(t-\sigma) \\
& =x_{i}\left(\left[\frac{t}{h}\right] h-\left[\frac{\sigma-1}{h}\right] h\right)-x_{i}\left(\left[\frac{t}{h}\right] h-\left[\frac{\sigma}{h}\right] h\right) .
\end{aligned}
$$

For $t \in[n h,(n+1) h], n \in Z_{0}^{+}$where $h$ is a fixed positive real number denoting a uniform discretisation step-size and $[r]$ denotes the integer part of the real number $r$. Clearly, for $t \in[n h,(n+1) h]$ we have $\left[\frac{t}{h}\right]=n$.

$$
\text { Let }\left[\frac{\tau}{h}\right]=\tau_{1},\left[\frac{\sigma-1}{h}\right]=\sigma^{0} \text { and }\left[\frac{\sigma}{h}\right]=\sigma^{1} \text {. }
$$

Therefore for each $i=1,2, \ldots \ldots . m$,

$$
y_{i}(t)=x_{i}(n h)+c_{i} x_{i}\left(\left(n-\tau_{1}\right) h\right)+p_{i}\left[x_{i}\left(\left(n-\sigma^{0}\right) h\right)-x_{i}\left(\left(n-\sigma^{1}\right) h\right)\right]+q_{i} \text {. }
$$

For convenience assume, $x_{i}(n h)=x_{i}(n)$ for each $i=1,2, \ldots \ldots . m$, we have

$$
y_{i}(t)=x_{i}(n)+c_{i} x_{i}\left(n-\tau_{1}\right)+p_{i}\left[x_{i}\left(n-\sigma^{0}\right)-x_{i}\left(n-\sigma^{1}\right)\right]+q_{i}=Z_{i}(n) .
$$

From (1) we have $\frac{d x_{i}(t)}{d t}=-a_{i} x_{i}(t)+\sum_{k=1}^{m} b_{i k} f_{k}\left(Z_{k}(n)\right)$,

then $\left[\frac{d x_{i}(t)}{d t}=a_{i} x_{i}(t)\right] e^{a_{i} t}=e^{a_{i} t} \sum_{k=1}^{m} b_{i k} f_{k}\left(Z_{k}(n)\right)$.

Simplifying we have $\frac{d}{d t}\left[x_{i}(t) e^{a_{i} t}\right]=e^{a_{i} t} \sum_{k=1}^{m} b_{i k} f_{k}\left(Z_{k}(n)\right)$.

Integrating over $(n h, t)$, where $t<(n+1) h$,

$$
x_{i}(t) e^{a_{i} t}-x_{i}(n h) e^{a_{i} n h}=\left[\frac{e^{a_{i} t}}{a_{i}}-\frac{e^{a_{i} n h}}{a_{i}}\right] \sum_{k=1}^{m} b_{i k} f_{k}\left(Z_{k}(n)\right) .
$$

Letting $t \rightarrow(n+1) h$, we can obtain

$$
x_{i}((n+1) h) e^{a_{i}(n+1) h}-x_{i}(n h) e^{a_{i} n h}=\left[\frac{e^{a_{i}(n+1) h}}{a_{i}}-\frac{e^{a_{i} n h}}{a_{i}}\right] \sum_{k=1}^{m} b_{i k} f_{k}\left(Z_{k}(n)\right) .
$$

After multiplication with $e^{-a_{i}(n+1) h}$ we can obtain,

$$
x_{i}((n+1) h)-x_{i}(n h) e^{-a_{i} h}=\left[\frac{1-e^{-a_{i} h}}{a_{i}}\right] \sum_{k=1}^{m} b_{i k} f_{k}\left(Z_{k}(n)\right) .
$$


For convenience we can assume $x_{i}(n h)=x_{i}(n)$,

$$
x_{i}((n+1))=x_{i}(n h) e^{-a_{i} h}+\phi_{i}(h) \sum_{k=1}^{m} b_{i k} f_{k}\left(Z_{k}(n)\right), \text { where } \phi_{i}(h)=\frac{1-e^{-a_{i} h}}{a_{i}} .
$$

Thus we can obtain for each $i=1,2, \ldots \ldots . m$,

$$
x_{i}(n+1)=\tilde{a}_{i} x_{i}(n)+\sum_{k=1}^{m} \tilde{b}_{i k} f_{k}\left(Z_{k}(n)\right)
$$

where

$$
Z_{i}(n)=x_{i}(n)+c_{i} x_{i}\left(n-\tau_{1}\right)+p_{i} x_{i}\left(n-\sigma^{0}\right)-p_{i} x_{i}\left(n-\sigma^{1}\right)+q_{i}
$$

and $\tilde{b}_{i k}=b_{i k} \phi_{i}(h), \tilde{a}_{i}=e^{-a_{i} h}, \quad i=1,2, \ldots m, k=1,2, \ldots m, \quad \tau_{1}=\left[\frac{\tau}{h}\right], \quad \sigma^{0}=\left[\frac{\sigma-1}{h}\right]$ and $\sigma^{1}=\left[\frac{\sigma}{h}\right]$

$h$ is a fixed positive real number denoting a uniform discretisation and $[r]$ denotes the integer part of the real number $r$.

Now the system (2) is discrete - time analogue of the continuous - time system (1).

Remark 1.1: Some differential equations with stable solution are unstable after discretisation using ordinary discretisation method or standard discretisation method rather solutions are stable by using proper approximation technique or non standard method, for example, the ordinary differential equation $y^{\prime}=-2 y$ has solution $y(t)=c e^{-2 T}$ then $y(t) \rightarrow 0$ as $t \rightarrow \infty$ which is a stable solution. Using ordinary discretisation, discrete equation is $y(n+1)+y(n)=0$, whose characteristic equation is $\lambda+1=0$, that is $\lambda=-1$ and the solution is $y(t)=c(-1)^{n}$ and $|\lambda|=1$. If the modulus of eigen values of the characteristic equation is less than 1 then the system is stable. But here $|\lambda|=1$. Therefore $y(n+1)+y(n)=0$ has unstable solution. Using proper approximation method, the discrete equation is $y(n+1)-e^{-2 h} y(n)=0$, whose characteristic equation is $\lambda-e^{-2 h}=0$, that is $\lambda=e^{-2 h}$, thus we have $|\lambda|=\left|e^{-2 h}\right|<1$. Therefore the system $y(n+1)-e^{-2 h} y(n)=0$ has stable solution. Due to this author felt that proper approximation technique is more relevant to obtain stable solution even after discretisation.

\section{Existence of unique equilibrium point}

Consider the system (3)

$$
Z_{i}(n)=x_{i}(n)+c_{i} x_{i}\left(n-\tau_{1}\right)+p_{i} x_{i}\left(n-\sigma^{0}\right)-p_{i} x_{i}\left(n-\sigma^{1}\right)+q_{i}, \quad i=1,2, \ldots m .
$$

Note that $Z_{i}(n)$ is governed by the neutral delay difference equation 


$$
\begin{aligned}
Z_{i}(n+1)= & \tilde{a}_{i} Z_{i}(n)+\sum_{k=1}^{m} \tilde{b}_{i k} f_{k}\left(Z_{k}(n)\right)+c_{i} \sum_{k=1}^{m} \tilde{b}_{i k} f_{k}\left(Z_{k}\left(n-\tau_{1}\right)\right) \\
& +p_{i} \sum_{k=1}^{m} \tilde{b}_{i k} f_{k}\left(Z_{k}\left(n-\sigma^{0}\right)\right)-p_{i} \sum_{k=1}^{m} \tilde{b}_{i k} f_{k}\left(Z_{k}\left(n-\sigma^{1}\right)\right)+I_{i},
\end{aligned}
$$

where $I_{i}=q_{i}-q_{i} \tilde{a}_{i}, i=1,2, \ldots m$.

In this section, consider the generalised discrete hysteretic neuron model equation (4) and guarantee the existence of a unique equilibrium to the system (4). Consider the space $C^{m}$ of all $m$-vectors of real numbers and let $\mid$. | denote the norm of an $m$-tuple $z=\left(z_{1}, z_{2}, \ldots ., z_{m}\right)$, defined by $|z|=\max _{1 \leq i \leq m}\left|z_{i}\right|$.

Now list the following hypothesis on the response functions $f_{k}, k=1,2, \ldots . m$ which will be needed in our subsequent discussion:

H1 Vectors $\eta, \varsigma \in C^{m}, f_{k}, k=1,2, \ldots . . m, L_{k}>0$ are constants, satisfies

$$
\left|f_{k}(\eta)-f_{k}(\varsigma)\right| \leq L_{k}|\eta-\varsigma|
$$

H2 $f_{k}, k=1,2, \ldots . m$ are continuous, complex valued functions and $f_{k}(0)=0$, $k=1,2, \ldots . . m$.

H3 There exists a constant $K_{k}>0, k=1,2, \ldots . m$ such that $\max _{z \in C^{m}}\left|f_{k}(z)\right|=K_{k}$.

From $\mathrm{H} 1$ and $\mathrm{H} 2$ we have

$$
\left|f_{k}(z)\right| \leq L_{k}|z|, \quad k=1,2, \ldots m
$$

If $z^{*}$ is the equilibrium point of the system (4) then $z^{*}$ satisfies the equation

$$
Z_{i}^{*}=\tilde{a}_{i} Z_{i}^{*}+\sum_{k=1}^{m} \tilde{b}_{i k} f_{k}\left(Z_{k}^{*}\right)+c_{i} \sum_{k=1}^{m} \tilde{b}_{i k} f_{k}\left(Z_{k}^{*}\right)+I_{i} .
$$

If $\mathrm{H} 1$ and $\mathrm{H} 2$ hold good that

$$
\max _{1 \leq i \leq m}\left|Z_{i}^{*}\right| \leq a_{0}\left|Z_{i}^{*}\right|+\max _{1 \leq i \leq m}\left|1+c_{i}\right| \sum_{k=1}^{m}\left|\tilde{b}_{i k}\right|\left|L_{k}\right|\left|Z_{i}^{*}\right|+|I|,
$$

where $a_{0}=\max _{1 \leq i \leq m} \tilde{a}_{i}$, and $|I|=\max _{1 \leq i \leq m}\left|I_{i}\right|$.

Thus $Z^{*}$ can also be represented through the following relation,

$$
\left|Z^{*}\right| \leq \frac{|I|}{\left(1-a_{0}-\max _{1 \leq i \leq m}\left|1+c_{i}\right| \sum_{k=1}^{m} L_{k}\left|\tilde{b}_{i k}\right|\right)},\left|Z^{*}\right|=\max _{1 \leq i \leq m}\left|Z_{i}^{*}\right|
$$

Provided

$$
0<a_{0}+\max _{1 \leq i \leq m}\left|1+c_{i}\right| \sum_{k=1}^{m} L_{k}\left|\tilde{b}_{i k}\right|<1 .
$$


Also, when $\mathrm{H} 3$ holds good if

$$
0<a_{0}=\max _{1 \leq i \leq m} \tilde{a}_{i}<1
$$

then $Z^{*}$ satisfies the estimate

$$
\left|Z^{*}\right| \leq \frac{\left.|I|+\max _{1 \leq i \leq m}\left|1+c_{i}\right| \sum_{k=1}^{m} L_{k}\left|\tilde{b}_{i k}\right|\right)}{1-a_{0}}
$$

We note that the inequalities (8) and (11) provide two sets of estimates for $Z^{*}$ which are valid under different conditions. From (7) we have

$$
Z_{i}^{*} \leq\left(1-\tilde{a}_{i}\right)^{-1}\left[\left(1+c_{i}\right) \sum_{k=1}^{m} \tilde{b}_{i k} f_{k}\left(Z_{k}^{*}\right)+I_{i}\right]
$$

We denote the minimum of the right hand estimates of $\left|Z^{*}\right|$ in the relations (9) and (10) by $\alpha$.

Let $S=\left\{z / z \in C^{m},|z|=\max _{1 \leq i \leq m}\left|z_{i}\right| \leq k\right\}$, for some $k$.

Then $Z^{*} \in S$ and hence $S$ is non empty compact set of $C^{m}$.

Define a mapping $T: S \rightarrow S$ by $T(z)=\left(T_{1}\left(z_{1}\right), T_{2}\left(z_{2}\right), \ldots \ldots, T_{m}\left(z_{m}\right)\right)$, where $T_{i}\left(z_{i}\right)=\left(1-\tilde{a}_{i}\right)^{-1}\left[\left(1+c_{i}\right) \sum_{k=1}^{m} \tilde{b}_{i k} f_{k}\left(z_{k}\right)+I_{i}\right], i=1,2, \ldots m$.

For any $z \in S$ we have $|z|=\max _{1 \leq i \leq m}\left|z_{i}\right|<k$

$$
\begin{aligned}
|T(z)| & =\max _{1 \leq i \leq m}\left|T_{i}\left(z_{i}\right)\right|=\max _{1 \leq i \leq m}\left|\left(1-\tilde{a}_{i}\right)^{-1}\left[\left(1+c_{i}\right) \sum_{k=1}^{m} \tilde{b}_{i k} f_{k}\left(z_{k}\right)+I_{i}\right]\right| \\
& \leq \max _{1 \leq i \leq m}\left(1-\tilde{a}_{i}\right)^{-1}\left[\left(1+\left|c_{i}\right|\right) \sum_{k=1}^{m}\left|\tilde{b}_{i k}\right|\left|L_{k}\right|\left|z_{k}\right|+\left|I_{i}\right|\right. \\
& \leq \max _{1 \leq i \leq m}\left(1-\tilde{a}_{i}\right)^{-1}\left[\left(1+\left|c_{i}\right|\right) k \sum_{k=1}^{m}\left|\tilde{b}_{i k}\right| L_{k}+\left|I_{i}\right|=\tilde{k} .\right.
\end{aligned}
$$

Then $T(z) \in S$ and hence $T(S) \subseteq S$.

Now for $Z, W \in S$,

$$
|T(Z)-T(W)| \leq \max _{1 \leq i \leq m}\left[\left(1-\tilde{a}_{i}\right)^{-1}\left[\left|1+c_{i}\right| \sum_{k=1}^{m}\left|\tilde{b}_{i k}\right|\left|f_{k}\left(z_{k}\right)-f_{k}\left(w_{k}\right)\right|\right]\right.
$$

Using H1, $|T(z)-T(w)| \leq \gamma|z-w|$ where $\gamma=\max _{1 \leq i \leq m}\left[\left(1-\tilde{a}_{i}\right)^{-1}\left[\left|1+c_{i}\right| \sum_{k=1}^{m}\left|\tilde{b}_{i k}\right| L_{k}\right]\right.$. If (9) holds we have $1-\tilde{a}_{i}>1-a_{0}>\max _{1 \leq i \leq m}\left|1+c_{i}\right| \sum_{k=1}^{m} L_{k}\left|\tilde{b}_{i k}\right|$ ) then we have $0<\gamma<1$. 
Hence $T$ is a contraction mapping. According to Banach's contraction mapping principle there exists a unique $Z^{*}$ in $S$, which satisfies $T\left(Z^{*}\right)=Z^{*}$, which will be required unique equilibrium pattern for the (4).

From the above discussion we can establish the following theorem.

Theorem 2.1: The system (4) possesses a unique equilibrium pattern if $\mathrm{H} 1$ and $\mathrm{H} 2$ hold and the inequality (9) is satisfied.

\section{Global exponential stability}

In this section, we consider the model (4) and established conditions for the global exponential stability of the equilibrium patterns. We shall tacitly assume that the network (4) possesses a unique equilibrium $Z^{*}=\left(Z_{1}^{*}, Z_{2}^{*}, \ldots, Z_{m}^{*}\right)$. Using the transformation $\tilde{Z}_{i}(n)=Z_{i}(n)-Z_{i}^{*}$ in equation (4) and utilising (7), we get for each $i=1,2, \ldots \ldots . m$,

$$
\begin{aligned}
\tilde{Z}_{i}(n+1)= & \tilde{a}_{i} \tilde{Z}_{i}(n)+\sum_{k=1}^{m} \tilde{b}_{i k} g_{k}\left(\tilde{Z}_{k}(n)\right)+c_{i} \sum_{k=1}^{m} \tilde{b}_{i k} g_{k}\left(\tilde{Z}_{k}\left(n-\tau_{1}\right)\right) \\
& +p_{i} \sum_{k=1}^{m} \tilde{b}_{i k}\left[g_{k}\left(\tilde{Z}_{k}\left(n-\sigma^{0}\right)\right)-g_{k}\left(\tilde{Z}_{k}\left(n-\sigma^{1}\right)\right)\right]
\end{aligned}
$$

in which $g_{k}\left(\tilde{Z}_{k}(n)\right)=f_{k}\left(\tilde{Z}_{k}(n)+Z_{k}^{*}\right)-f_{k}\left(Z_{k}^{*}\right), k=1,2, \ldots m$

Redesignating $\tilde{Z}(n)$ as $z(n)$, we finally obtain

$$
\begin{aligned}
z_{i}(n+1)= & \tilde{a}_{i} z_{i}(n)+\sum_{k=1}^{m} \tilde{b}_{i k} g_{k}\left(z_{k}(n)\right)+c_{i} \sum_{k=1}^{m} \tilde{b}_{i k} g_{k}\left(z_{k}\left(n-\tau_{1}\right)\right) \\
& +p_{i} \sum_{k=1}^{m} \tilde{b}_{i k}\left[g_{k}\left(z_{k}\left(n-\sigma^{0}\right)\right)-g_{k}\left(z_{k}\left(n-\sigma^{1}\right)\right)\right]
\end{aligned}
$$

Clearly stability of $z^{*}$ for the system (4) is equivalent to the stability of the trivial solution for the system (13) and if $\mathrm{H} 1 \mathrm{holds}$, then we have

$$
\mid g_{k}\left(z(n)\left|\leq L_{k}\right| z(n) \mid, k=1,2, \ldots m .\right.
$$

Definition 3.1 (Zhang et al., 2006): The trivial solution of equation (13) is said to be globally exponentially stable if for any solution $z_{i}\left(n, z_{i}\left(n_{0}\right)\right)$, with the initial condition $z_{i}\left(n_{0}\right)=z_{i}\left(0, n_{0}\right)$, there exist constants $\rho \in(0,1)$ and $M \geq 1$ such that

$$
\sum_{i=1}^{m}\left|z_{i}(n)\right|^{2} \leq M\|\phi\|^{2} \quad \rho^{n}, n \geq n_{0}
$$

where $\|\phi\|^{2}=\max \left\{z_{i}(0), \sum_{i=1}^{m} z_{i}^{2}(0), \sum_{i=1}^{m} z_{i}^{2}(-1), \ldots \ldots \sum_{i=1}^{m} z_{i}^{2}\left(n_{0}\right)\right\}$.

Lemma 3.1 (Liz and Ferreiro, 2002): Let $r>0$ be a natural number, and let $\{Z(n)\}_{n \geq-r}$ be a sequence of real numbers satisfying the following inequality 


$$
\Delta Z(n) \leq-p Z(n)+q \max \{Z(n), Z(n-1), \ldots Z(n-r)\},
$$

where $n \geq 0$. If $0<q<p \leq 1$ then there exists a constant $\lambda_{0} \in(0,1)$ such that

$$
Z(n) \leq \max \{Z(0), Z(-1), \ldots Z(-r)\} \lambda_{0}^{n}, n \geq 0 .
$$

Moreover $\lambda_{0}$ can be chosen as the smallest root in the interval $(0,1)$ of the equation

$$
\lambda^{r+1}+(p-1) \lambda^{r}-q=0 .
$$

Now we state and prove the main result in the foregoing sections.

Theorem 3.1: Assume that the condition $\mathrm{H} 1$ and (9) holds and that there exist positive numbers $\eta_{i}, \delta_{i}, i=1,2, \ldots, 21$. If

$$
\begin{gathered}
\tilde{q}=4 \beta L_{\max }^{2}\left(\lambda_{\max }\left(c^{\mathrm{T}} c\right)+\lambda_{\max }\left(p^{\mathrm{T}} p\right)\right)\left[\lambda_{\max }\left(A^{\mathrm{T}} A\right)+\lambda_{\max }\left(B^{\mathrm{T}} B\right)\right] \\
<\tilde{p}=\max _{1 \leq i \leq m}\left[1-\beta\left|\tilde{a}_{i}\right|^{2}-2 \beta\left|L_{i}\right|^{2}\left(\lambda_{\max }\left(A^{\mathrm{T}} A\right)+\lambda_{\max }\left(B^{\mathrm{T}} B\right)\right)\right],
\end{gathered}
$$

where $\lambda_{\max }(A)$ the largest eigen value of a matrix A, $L_{\max }=\max _{1 \leq i \leq m}\left\{L_{i}\right\}, L=\operatorname{diag}\left\{L_{1}, L_{2}\right.$, $\left.\ldots . . L_{m}\right\}$ and

$$
\beta=\max \left\{\begin{array}{l}
\left(1+\eta_{1}+\eta_{2}+\eta_{3}+\eta_{4}+\eta_{5}+\eta_{6}\right),\left(1+\eta_{1}^{-1}+\eta_{7}+\eta_{8}+\eta_{9}+\eta_{10}+\eta_{11}\right), \\
\left(1+\eta_{2}^{-1}+\eta_{7}^{-1}+\eta_{12}+\eta_{13}+\eta_{14}+\eta_{15}\right),\left(1+\eta_{3}^{-1}+\eta_{8}^{-1}+\eta_{12}^{-1}+\eta_{16}^{-1}+\eta_{17}+\eta_{18}\right), \\
\left(1+\eta_{4}^{-1}+\eta_{9}^{-1}+\eta_{13}^{-1}+\eta_{16}+\eta_{19}+\eta_{20}\right),\left(1+\eta_{5}^{-1}+\eta_{10}^{-1}+\eta_{14}^{-1}+\eta_{17}^{-1}+\eta_{19}^{-1}+\eta_{21}\right), \\
\left(1+\eta_{6}^{-1}+\eta_{11}^{-1}+\eta_{15}^{-1}+\eta_{18}^{-1}+\eta_{20}^{-1}++\eta_{21}^{-1}\right),\left(1+\delta_{1}+\delta_{2}+\delta_{3}+\delta_{4}+\delta_{5}+\delta_{6}\right), \\
\left(1+\delta_{1}^{-1}+\delta_{7}+\delta_{8}+\delta_{9}+\delta_{10}+\delta_{11}\right),\left(1+\delta_{2}^{-1}+\delta_{7}^{-1}+\delta_{12}+\delta_{13}+\delta_{14}+\delta_{15}\right), \\
\left(1+\delta_{3}^{-1}+\delta_{8}^{-1}+\delta_{12}^{-1}+\delta_{16}^{-1}+\delta_{17}+\delta_{18}\right),\left(1+\delta_{4}^{-1}+\delta_{9}^{-1}+\delta_{13}^{-1}+\delta_{16}+\delta_{19}+\delta_{20}\right), \\
\left(1+\delta_{5}^{-1}+\delta_{10}^{-1}+\delta_{14}^{-1}+\delta_{17}^{-1}+\delta_{19}^{-1}+\delta_{21}\right),\left(1+\delta_{6}^{-1}+\delta_{11}^{-1}+\delta_{15}^{-1}+\delta_{18}^{-1}+\delta_{20}^{-1}+\delta_{21}^{-1}\right),
\end{array}\right.
$$

Then the equilibrium point of (13) is globally exponentially stable.

Proof: System (13) may be put in the following equivalent form

$$
\begin{aligned}
z(n+1)= & a z(n)+\operatorname{Mg}(z(n))+c \operatorname{Mg}\left(z\left(n-\tau_{1}\right)\right) \\
& +p \operatorname{Mg}\left[g\left(z\left(n-\sigma^{0}\right)\right)-g\left(z\left(n-\sigma^{1}\right)\right)\right]
\end{aligned}
$$

$c=\operatorname{diag}\left\{c_{1}, c_{2}\right.$, $\left.c_{m}\right\}, \quad \tilde{a}=\operatorname{diag}\left\{\tilde{a}_{1}, \tilde{a}_{2}\right.$ $\left.\tilde{a}_{m}\right\}, \quad p=\operatorname{diag}\left\{p_{1}, p_{2}\right.$,

$M=\left[\tilde{b}_{i k}\right]$ and $g$ is the $m$-vector with components $g_{k}, k=1,2, \ldots m$.

Let $U(n)$ and $V(n)$ denote the real and imaginary parts of $z(n)$ so that $z(n)=U(n)+$ $j V(n)$, in which $j=\sqrt{-1}$. Also, we express the other complex quantities in (17) by separating the real and imaginary parts $M=A+j B, g(z(n)=h(z(n))+j k(z(n))$. Here all 
the quantities $A, B, U, V, h$ and $k$ are real. Now separating the real and imaginary parts in (17), we obtain

$$
\begin{aligned}
U(n+1)= & \tilde{a} U(n)+A h(z(n))-B k\left(z(n)+c A h\left(z\left(n-\tau_{1}\right)\right)-c B k\left(z\left(n-\tau_{1}\right)\right.\right. \\
& +p A\left[h\left(z\left(n-\sigma^{0}\right)\right)-h\left(z\left(n-\sigma^{1}\right)\right)\right] \\
& -p B\left[k\left(z\left(n-\sigma^{0}\right)\right)-k\left(z\left(n-\sigma^{1}\right)\right)\right], \\
V(n+1)= & \tilde{a} V(n)+B h(z(n))+A k\left(z(n)+c A k\left(z\left(n-\tau_{1}\right)\right)+c B h\left(z\left(n-\tau_{1}\right)\right.\right. \\
& +p A\left[k\left(z\left(n-\sigma^{0}\right)\right)-k\left(z\left(n-\sigma^{1}\right)\right)\right] \\
& +p B\left[h\left(z\left(n-\sigma^{0}\right)\right)-h\left(z\left(n-\sigma^{1}\right)\right)\right] .
\end{aligned}
$$

Consider the following function

$$
\begin{aligned}
W(n) & =|Z(n)|^{2}=\bar{Z}^{T}(n) Z(n) \\
& =\sum_{i=1}^{m} U_{i}^{2}(n)+\sum_{i=1}^{m} V_{i}^{2}(n) \\
& =U^{T}(n) U(n)+V^{T}(n) V(n),
\end{aligned}
$$

in which $T$ denotes the transpose of a vector, so that

$$
W(n+1)=U^{T}(n+1) U(n+1)+V^{T}(n+1) V(n+1) .
$$

We now compute the first term on the right hand side of (18) as follows

$$
\begin{aligned}
& U^{T}(n+1) U(n+1) \\
= & \left(U^{T}(n) \tilde{a}^{T}+h^{T}(z(n)) A^{T}-k^{T}\left(z(n) B^{T}+h^{T}\left(z\left(n-\tau_{1}\right)\right) A^{T} c^{T}\right.\right. \\
& -k^{T}\left(z\left(n-\tau_{1}\right) B^{T} c^{T}\right. \\
& +\left[h^{T}\left(z\left(n-\sigma^{0}\right)\right)-h^{T}\left(z\left(n-\sigma^{1}\right)\right)\right] A^{T} p^{T} \\
& \left.-\left[k^{T}\left(z\left(n-\sigma^{0}\right)\right)-k^{T}\left(z\left(n-\sigma^{1}\right)\right)\right] B^{T} p^{T}\right) \\
& \times\left(\tilde{a} U(n)+A h(z(n))-B k\left(z(n)+c A h\left(z\left(n-\tau_{1}\right)\right)-c B k\left(z\left(n-\tau_{1}\right)\right.\right.\right. \\
& +p A\left[h\left(z\left(n-\sigma^{0}\right)\right)-h\left(z\left(n-\sigma^{1}\right)\right)\right] \\
& \left.-p B\left[k\left(z\left(n-\sigma^{0}\right)\right)-k\left(z\left(n-\sigma^{1}\right)\right)\right]\right)
\end{aligned}
$$


For convenience write

$$
\begin{aligned}
& \tilde{h}(z(n-\alpha))=\left[h\left(z\left(n-\sigma^{0}\right)\right)-h\left(z\left(n-\sigma^{1}\right)\right)\right] \\
& \text { and } \tilde{k}(z(n-\alpha))=\left[k\left(z\left(n-\sigma^{0}\right)\right)-k\left(z\left(n-\sigma^{1}\right)\right)\right] .
\end{aligned}
$$

Clearly if H1 holds and from (14) we have $\left|h_{k}(z(n))\right| \leq L_{k}|z(n)|$ and $\left|k_{k}(z(n))\right|$ $\leq L_{k}|z(n)|, k=1,2, \ldots m$.

And hence we can represent

$$
|\tilde{h}(z(n-\alpha))| \leq L_{\max }|z(n-\alpha)| \text {, and }|\tilde{k}(z(n-\alpha))| \leq L_{\max }|z(n-\alpha)| \text {, }
$$

where $L_{\max }=\max _{1 \leq i \leq m}\left\{L_{i}\right\}$.

Using (19) and simplifying we have

$$
\begin{aligned}
& U^{T}(n+1) U(n+1) \\
&=U^{T}(n) \tilde{a}^{T}\left(\tilde{a} U(n)+A h(z(n))-B k\left(z(n)+c A h\left(z\left(n-\tau_{1}\right)\right)\right.\right. \\
&-c B k\left(z\left(n-\tau_{1}\right)+p A \tilde{h}(z(n-\alpha))-p B \tilde{k}(z(n-\alpha))\right. \\
&+h^{T}(z(n)) A^{T}(\tilde{a} U(n)+A h(z(n))-B k(z(n) \\
&+c A h\left(z\left(n-\tau_{1}\right)\right)-c B k\left(z\left(n-\tau_{1}\right)+p A \tilde{h}(z(n-\alpha))\right. \\
&-p B \tilde{k}(z(n-\alpha))-k^{T}\left(z(n) B^{T}(\tilde{a} U(n)+A h(z(n))-B k(z(n)\right. \\
&+c A h\left(z\left(n-\tau_{1}\right)\right)-c B k\left(z\left(n-\tau_{1}\right)-B k(z(n)+p A \tilde{h}(z(n-\alpha))\right. \\
&-p B \tilde{k}(z(n-\alpha))+h^{T}\left(z\left(n-\tau_{1}\right)\right) A^{T} c^{T}(\tilde{a} U(n) \\
&+A h(z(n))+c A h\left(z\left(n-\tau_{1}\right)\right)-c B k\left(z\left(n-\tau_{1}\right)+p A \tilde{h}(z(n-\alpha))\right. \\
&-p B \tilde{k}(z(n-\alpha))-k^{T}\left(z\left(n-\tau_{1}\right) B^{T} c^{T}(\tilde{a} U(n)\right. \\
&+A h(z(n))-B k\left(z(n)+c A h\left(z\left(n-\tau_{1}\right)\right)-c B k\left(z\left(n-\tau_{1}\right)\right.\right. \\
&+p A \tilde{h}(z(n-\alpha))-p B \tilde{k}(z(n-\alpha))+\tilde{h}^{T}(z(n-\alpha)) A^{T} p^{T}(\tilde{a} U(n) \\
&+A h(z(n))-B k\left(z(n)+c A h\left(z\left(n-\tau_{1}\right)\right)-c B k\left(z\left(n-\tau_{1}\right)\right.\right. \\
&+p A \tilde{h}(z(n-\alpha))-p B \tilde{k}(z(n-\alpha)) \\
&-\tilde{k}^{T}(z(n-\alpha)) B^{T} p^{T}(\tilde{a} U(n)+A h(z(n))-B k(z(n) \\
&+c A h\left(z\left(n-\tau_{1}\right)\right)-c B k\left(z\left(n-\tau_{1}\right)\right. \\
&+p A \tilde{h}(z(n-\alpha))-p B \tilde{k}(z(n-\alpha)) . \\
&+z(n)
\end{aligned}
$$

Rearranging the terms we obtain 


$$
\begin{aligned}
& U^{T}(n+1) U(n+1) \\
& =|\tilde{a}|^{2} U^{T}(n) U(n)+\left[U^{T}(n) \tilde{a}^{T} A h(z(n))+h^{T}(z(n)) A^{T} \tilde{a} U(n)\right] \\
& -\left[U^{T}(n) \tilde{a}^{T} B k(z(n))+\tilde{a} U(n) k^{T}(z(n)) B^{T}\right] \\
& +\left[U^{T}(n) \tilde{a}^{T} c A h\left(z\left(n-\tau_{1}\right)\right)+h^{T}\left(z\left(n-\tau_{1}\right)\right) A^{T} c^{T} \tilde{a} U(n)\right] \\
& -\left[U^{T}(n) \tilde{a}^{T} c B k\left(z\left(n-\tau_{1}\right)\right)+k^{T}\left(z\left(n-\tau_{1}\right)\right) B^{T} c^{T} \tilde{a} U(n)\right] \\
& +\left[U^{T}(n) \tilde{a}^{T} p A \tilde{h}(z(n-\alpha))+\tilde{h}^{T}(z(n-\alpha)) A^{T} p^{T} \tilde{a} U(n)\right] \\
& -\left[U^{T}(n) \tilde{a}^{T} p B \tilde{k}(z(n-\alpha))+\tilde{k}^{T}(z(n-\alpha)) B^{T} p^{T} \tilde{a} U(n)\right] \\
& +h^{T}(z(n)) A^{T} A h(z(n)) \\
& -\left[h^{T}(z(n)) h^{T} B k(z(n))+k^{T}(z(n)) B^{T} A h(z(n))\right] \\
& +\left[h^{T}(z(n)) A^{T} c A h\left(z\left(n-\tau_{1}\right)\right)+h^{T}\left(z\left(n-\tau_{1}\right)\right) A^{T} c^{T} A h(z(n))\right] \\
& -\left[h^{T}(z(n)) A^{T} c B k\left(z\left(n-\tau_{1}\right)\right)+k^{T}\left(z\left(n-\tau_{1}\right)\right) B^{T} c^{T} A h(z(n))\right] \\
& +\left[h^{T}(z(n)) A^{T} p A h_{1}(z(n-\alpha))+\tilde{h}^{T}(z(n-\alpha)) A^{T} p^{T} A h(z(n))\right] \\
& -\left[h^{T}(z(n)) A^{T} p B k_{1}(z(n-\alpha))+\tilde{k}^{T}(z(n-\alpha)) B^{T} p^{T} A h(z(n))\right] \\
& +k^{T}(z(n)) B^{T} B k(z(n)) \\
& -\left[k^{T}(z(n)) B^{T} c A h\left(z\left(n-\tau_{1}\right)\right)+h^{T}\left(z\left(n-\tau_{1}\right)\right) A^{T} c^{T} B k(z(n))\right] \\
& +\left[k^{T}(z(n)) B^{T} c B k\left(z\left(n-\tau_{1}\right)\right)+k^{T}\left(z\left(n-\tau_{1}\right)\right) B^{T} c^{T} B k(z(n))\right] \\
& -\left[k^{T}(z(n)) B^{T} p A \tilde{h}(z(n-\alpha))+\tilde{h}^{T}(z(n-\alpha)) A^{T} p^{T} B k(z(n))\right] \\
& +\left[k^{T}(z(n)) B^{T} p B \tilde{k}(z(n-\alpha))+\tilde{k}^{T}(z(n-\alpha)) B^{T} B k(z(n))\right] \\
& +h^{T}\left(z\left(n-\tau_{1}\right)\right) A^{T} c^{T} c A h\left(z\left(n-\tau_{1}\right)\right) \\
& -\left[h^{T}\left(z\left(n-\tau_{1}\right)\right) A^{T} c B k\left(z\left(n-\tau_{1}\right)\right)+k^{T}\left(z\left(n-\tau_{1}\right)\right) B^{T} c^{T} c A h\left(z\left(n-\tau_{1}\right)\right)\right] \\
& +\left[h^{T}\left(z\left(n-\tau_{1}\right)\right) A^{T} c^{T} p A \tilde{h}(z(n-\alpha))+\tilde{h}^{T}(z(n-\alpha)) A^{T} p^{T} c A h\left(z\left(n-\tau_{1}\right)\right)\right] \\
& -\left[h^{T}\left(z\left(n-\tau_{1}\right)\right) B^{T} c^{T} p B \tilde{k}(z(n-\alpha))+\tilde{k}^{T}(z(n-\alpha)) B^{T} p^{T} c A h\left(z\left(n-\tau_{1}\right)\right)\right] \\
& +k^{T}\left(z\left(n-\tau_{1}\right)\right) B^{T} c^{T} c B k\left(z\left(n-\tau_{1}\right)\right) \\
& -\left[k^{T}\left(z\left(n-\tau_{1}\right)\right) B^{T} c^{T} p A \tilde{h}(z(n-\alpha))+\tilde{h}^{T}(z(n-\alpha)) A^{T} p^{T} c k\left(z\left(n-\tau_{1}\right)\right)\right] \\
& +\left[k^{T}\left(z\left(n-\tau_{1}\right)\right) B^{T} c^{T} p B \tilde{k}(z(n-\alpha))+\tilde{k}^{T}(z(n-\alpha)) B^{T} p^{T} c k\left(z\left(n-\tau_{1}\right)\right)\right] \\
& +\tilde{h}^{T}(z(n-\alpha)) A^{T} p^{T} p A \tilde{h}(z(n-\alpha)) \\
& +\left[\tilde{h}^{T}(z(n-\alpha)) A^{T} p^{T} p B \tilde{k}(z(n-\alpha))+\tilde{k}^{T}(z(n-\alpha)) B^{T} p^{T} p A \tilde{h}(z(n-\alpha))\right] \\
& +\tilde{k}^{T}(z(n-\alpha)) B^{T} p^{T} p B \tilde{k}(z(n-\alpha)) .
\end{aligned}
$$


Using the inequalities $x^{T} y+y^{T} x \leq \eta x^{T} x+\eta^{-1} y^{T} y$ for any vectors $x, y \in R^{m}$ and $\eta>0$, for the term in the square brackets on the right hand side of (21) we obtain

$$
\begin{aligned}
& U^{T}(n+1) U(n+1) \\
& \leq|\tilde{a}|^{2} U^{T}(n) U(n)+\left[\eta_{1}|\tilde{a}|^{2} U^{T}(n) U(n)+\eta_{1}^{-1} h^{T}(z(n)) A^{T} A h(z(n))\right] \\
& -\left[\eta_{2}|\tilde{a}|^{2} U^{T}(n) U(n)+\eta_{2}^{-1} h^{T}(z(n)) B^{T} B k(z(n))\right] \\
& +\left[\eta_{3}|\tilde{a}|^{2} U^{T}(n) U(n)+\eta_{3}^{-1} h^{T}\left(z\left(n-\tau_{1}\right)\right) A^{T} c^{T} c A h\left(z\left(n-\tau_{1}\right)\right)\right] \\
& -\left[\eta_{4}|\tilde{a}|^{2} U^{T}(n) U(n)+\eta_{4}^{-1} k^{T}\left(z\left(n-\tau_{1}\right)\right) B^{T} c^{T} c B k\left(z\left(n-\tau_{1}\right)\right)\right] \\
& +\left[\eta_{5}|\tilde{a}|^{2} U^{T}(n) U(n)+\eta_{5}^{-1} \tilde{h}^{T}(z(n-\alpha)) A^{T} p^{T} p A \tilde{h}(z(n-\alpha))\right] \\
& -\left[\eta_{6}|\tilde{a}|^{2} U^{T}(n) U(n)+\eta_{6}^{-1} \tilde{k}^{T}(z(n-\alpha)) B^{T} p^{T} p B \tilde{k}(z(n-\alpha))\right] \\
& +h^{T}(z(n)) A^{T} A h(z(n))+k^{T}(z(n)) B^{T} B k(z(n)) \\
& -\left[\eta_{7} h^{T}(z(n)) A^{T} A h(z(n))+\eta_{7}^{-1} k^{T}(z(n)) B^{T} B k(z(n))\right] \\
& +\left[\eta_{8} h^{T}(z(n)) A^{T} A h(z(n))+\eta_{8}^{-1} h^{T}\left(z\left(n-\tau_{1}\right)\right) A^{T} c^{T} c A h\left(z\left(n-\tau_{1}\right)\right)\right] \\
& -\left[\eta_{9} h^{T}(z(n)) A^{T} A h(z(n))+\eta_{9}^{-1} k^{T}\left(z\left(n-\tau_{1}\right)\right) B^{T} c^{T} c B k\left(z\left(n-\tau_{1}\right)\right)\right] \\
& +\left[\eta_{10} h^{T}(z(n)) A^{T} A h(z(n))+\eta_{10}^{-1} \tilde{h}^{T}(z(n-\alpha)) A^{T} p^{T} p A \tilde{h}(z(n-\alpha))\right] \\
& -\left[\eta_{11} h^{T}(z(n)) A^{T} A h(z(n))+\eta_{11}^{-1} \tilde{k}^{T}(z(n-\alpha)) B^{T} p^{T} p B \tilde{k}(z(n-\alpha))\right] \\
& -\left[\eta_{12} k^{T}(z(n)) B^{T} B k(z(n))+\eta_{12}^{-1} h^{T}\left(z\left(n-\tau_{1}\right)\right) A^{T} c^{T} c A h\left(z\left(n-\tau_{1}\right)\right)\right] \\
& +\left[\eta_{13} k^{T}(z(n)) B^{T} B k(z(n))+\eta_{13}^{-1} k^{T}\left(z\left(n-\tau_{1}\right)\right) B^{T} c^{T} c B k\left(z\left(n-\tau_{1}\right)\right)\right] \\
& -\left[\eta_{14} k^{T}(z(n)) B^{T} B k(z(n))+\eta_{14}^{-1} \tilde{h}^{T}(z(n-\alpha)) A^{T} p^{T} p A \tilde{h}(z(n-\alpha))\right] \\
& +\left[\eta_{15} k^{T}(z(n)) B^{T} B k(z(n))+\eta_{15}^{-1} \tilde{k}^{T}(z(n-\alpha)) B^{T} p^{T} p B \tilde{k}(z(n-\alpha))\right] \\
& +h^{T}\left(z\left(n-\tau_{1}\right)\right) A^{T} c^{T} c A h\left(z\left(n-\tau_{1}\right)\right)+\tilde{k}^{T}(z(n-\alpha)) B^{T} p^{T} p B \tilde{k}(z(n-\alpha)) \\
& -\left[\eta_{16} h^{T}\left(z\left(n-\tau_{1}\right)\right) A^{T} c^{T} c A h\left(z\left(n-\tau_{1}\right)\right)+\eta_{16}^{-1} k^{T}\left(z\left(n-\tau_{1}\right)\right) B^{T} c^{T} c B k\left(z\left(n-\tau_{1}\right)\right)\right] \\
& +\left[\eta_{17} h^{T}\left(z\left(n-\tau_{1}\right)\right) A^{T} c^{T} c A h\left(z\left(n-\tau_{1}\right)\right)+\eta_{17}^{-1} \tilde{h}^{T}(z(n-\alpha)) A^{T} p^{T} p A \tilde{h}(z(n-\alpha))\right] \\
& -\left[\eta_{18} h^{T}\left(z\left(n-\tau_{1}\right)\right) A^{T} c^{T} c A h\left(z\left(n-\tau_{1}\right)\right)+\eta_{17}^{-1} \tilde{k}^{T}(z(n-\alpha)) B^{T} p^{T} p B \tilde{k}(z(n-\alpha))\right] \\
& +k^{T}\left(z\left(n-\tau_{1}\right)\right) B^{T} c^{T} c B k\left(z\left(n-\tau_{1}\right)\right)+\tilde{h}^{T}(z(n-\alpha)) A^{T} p^{T} p A \tilde{h}(z(n-\alpha)) \\
& -\left[\eta_{19} k^{T}\left(z\left(n-\tau_{1}\right)\right) B^{T} c^{T} c k\left(z\left(n-\tau_{1}\right)\right)+\eta_{19}^{-1} \tilde{h}^{T}(z(n-\alpha)) A^{T} p^{T} p A \tilde{h}(z(n-\alpha))\right] \\
& +\left[\eta_{20} k^{T}\left(z\left(n-\tau_{1}\right)\right) B^{T} c^{T} c k\left(z\left(n-\tau_{1}\right)\right)+\eta_{20}^{-1} \tilde{k}^{T}(z(n-\alpha)) B^{T} p^{T} p B \tilde{k}(z(n-\alpha))\right] \\
& +\left[\eta_{21} \tilde{h}^{T}(z(n-\alpha)) A^{T} p^{T} p A \tilde{h}(z(n-\alpha))+\eta_{20}^{-1} \tilde{k}^{T}(z(n-\alpha)) B^{T} p^{T} p B \tilde{k}(z(n-\alpha))\right],
\end{aligned}
$$


Simplifying we obtain,

$$
\begin{aligned}
& U^{T}(n+1) U(n+1) \\
& \leq|\tilde{a}|^{2}\left[1+\eta_{1}+\eta_{2}+\eta_{3}+\eta_{4}+\eta_{5}+\eta_{6}\right] U^{T}(n) U(n) \\
&+ {\left[1+\eta_{1}^{-1}+\eta_{7}+\eta_{8}+\eta_{9}+\eta_{10}+\eta_{11}\right] h^{T}(z(n)) \lambda_{\max }\left(A^{T} A\right) h(z(n)) } \\
&+ {\left[1+\eta_{2}^{-1}+\eta_{7}^{-1}+\eta_{12}+\eta_{13}+\eta_{14}+\eta_{15}\right] k^{T}(z(n)) \lambda_{\max }\left(B^{T} B\right) k(z(n)) } \\
&+|c|^{2}\left[1+\eta_{3}^{-1}+\eta_{8}^{-1}+\eta_{12}^{-1}+\eta_{16}^{-1}+\eta_{17}+\eta_{18}\right] \\
& h^{T}\left(z\left(n-\tau_{1}\right)\right) \lambda_{\max }\left(A^{T} A\right) h\left(z\left(n-\tau_{1}\right)\right) \\
&+|c|^{2}\left[1+\eta_{4}^{-1}+\eta_{9}^{-1}+\eta_{13}^{-1}+\eta_{16}+\eta_{19}+\eta_{20}\right] \\
& k^{T}\left(z\left(n-\tau_{1}\right)\right) \lambda_{\max }\left(B^{T} B\right) k\left(z\left(n-\tau_{1}\right)\right) \\
&+|p|^{2}\left[1+\eta_{5}^{-1}+\eta_{10}^{-1}+\eta_{14}^{-1}+\eta_{17}^{-1}+\eta_{19}^{-1}+\eta_{21}\right] \\
& \tilde{h}^{T}(z(n-\alpha)) \lambda_{\max }\left(A^{T} A\right) \tilde{h}(z(n-\alpha)) \\
&+|p|^{2}\left[1+\eta_{6}^{-1}+\eta_{11}^{-1}+\eta_{15}^{-1}+\eta_{18}^{-1}+\eta_{20}^{-1}+\eta_{21}^{-1}\right] \\
& \tilde{k}^{T}(z(n-\alpha)) \lambda_{\max }\left(B^{T} B\right) \tilde{k}(z(n-\alpha))
\end{aligned}
$$

Also adopting the similar procedure for computing $V^{T}(n+1) V(n+1)$ we obtain

$$
\begin{aligned}
& V^{T}(n+1) V(n+1) \\
& \leq|\tilde{a}|^{2}\left[1+\delta_{1}+\delta_{2}+\delta_{3}+\delta_{4}+\delta_{5}+\delta_{6}\right] V^{T}(n) V(n) \\
&+ {\left[1+\delta_{1}^{-1}+\delta_{7}+\delta_{8}+\delta_{9}+\delta_{10}+\delta_{11}\right] } \\
& h^{T}(z(n)) \lambda_{\max }\left(B^{T} B\right) h(z(n)) \\
&+ {\left[1+\delta_{2}^{-1}+\delta_{7}^{-1}+\delta_{12}+\delta_{13}+\delta_{14}+\delta_{15}\right] } \\
& k^{T}(z(n)) \lambda_{\max }\left(A^{T} A\right) k(z(n)) \\
&+|c|^{2}\left[1+\delta_{3}^{-1}+\delta_{8}^{-1}+\delta_{12}^{-1}+\delta_{16}^{-1}+\delta_{17}+\delta_{18}\right] \\
& h^{T}\left(z\left(n-\tau_{1}\right)\right) \lambda_{\max }\left(B^{T} B\right) h\left(z\left(n-\tau_{1}\right)\right) \\
&+|c|^{2}\left[1+\delta_{4}^{-1}+\delta_{9}^{-1}+\delta_{13}^{-1}+\delta_{16}+\delta_{19}+\delta_{20}\right] \\
& k^{T}\left(z\left(n-\tau_{1}\right)\right) \lambda_{\max }\left(A^{T} A\right) k\left(z\left(n-\tau_{1}\right)\right) \\
&+|p|^{2}\left[1+\delta_{5}^{-1}+\delta_{10}^{-1}+\delta_{14}^{-1}+\delta_{17}^{-1}+\delta_{19}^{-1}+\delta_{21}\right] \\
& \tilde{h}^{T}(z(n-\alpha)) \lambda_{\max }\left(B^{T} B\right) \tilde{h}(z(n-\alpha)) \\
&+|p|^{2}\left[1+\delta_{6}^{-1}+\delta_{11}^{-1}+\delta_{15}^{-1}+\delta_{18}^{-1}+\delta_{20}^{-1}+\delta_{21}^{-1}\right] \\
& \tilde{k}^{T}(z(n-\alpha)) \lambda_{\max }\left(A^{T} A\right) \tilde{k}(z(n-\alpha)) . \\
&+0 \delta_{1}>0, i=1,2, \ldots 1 .
\end{aligned}
$$

for $\delta_{i}>0, i=1,2, \ldots 21$. 
From (22) and (23), we have

$$
\begin{aligned}
& U^{T}(n+1) U(n+1) \\
& \leq|\tilde{a}|^{2}\left\{\left[1+\eta_{1}+\eta_{2}+\eta_{3}+\eta_{4}+\eta_{5}+\eta_{6}\right] U^{T}(n) U(n)\right. \\
& \left.+\left[1+\delta_{1}+\delta_{2}+\delta_{3}+\delta_{4}+\delta_{5}+\delta_{6}\right] V^{T}(n) V(n)\right\} \\
& +h^{T}(z(n))\left\{\left[1+\eta_{1}^{-1}+\eta_{7}+\eta_{8}+\eta_{9}+\eta_{10}+\eta_{11}\right] \lambda_{\max }\left(A^{T} A\right)\right. \\
& \left.+\left[1+\delta_{1}^{-1}+\delta_{7}+\delta_{8}+\delta_{9}+\delta_{10}+\delta_{11}\right] \lambda_{\max }\left(B^{T} B\right)\right\} h(z(n)) \\
& +k^{T}(z(n))\left\{\left[1+\eta_{2}^{-1}+\eta_{7}^{-1}+\eta_{12}+\eta_{13}+\eta_{14}+\eta_{15}\right] \lambda_{\max }\left(B^{T} B\right)\right. \\
& \left.+\left[1+\delta_{2}^{-1}+\delta_{7}^{-1}+\delta_{12}+\delta_{13}+\delta_{14}+\delta_{15}\right] \lambda_{\max }\left(A^{T} A\right)\right\} k(z(n)) \\
& +|c|^{2}\left\{\left[1+\eta_{3}^{-1}+\eta_{8}^{-1}+\eta_{12}^{-1}+\eta_{16}^{-1}+\eta_{17}+\eta_{18}\right] h^{T}\left(z\left(n-\tau_{1}\right)\right) \lambda_{\max }\left(A^{T} A\right) h\left(z\left(n-\tau_{1}\right)\right)\right. \\
& \left.+\left[1+\delta_{3}^{-1}+\delta_{8}^{-1}+\delta_{12}^{-1}+\delta_{16}^{-1}+\delta_{17}+\delta_{18}\right] h^{T}\left(z\left(n-\tau_{1}\right)\right) \lambda_{\max }\left(B^{T} B\right) h\left(z\left(n-\tau_{1}\right)\right)\right\} \\
& +|c|^{2}\left\{\left[1+\eta_{4}^{-1}+\eta_{9}^{-1}+\eta_{13}^{-1}+\eta_{16}+\eta_{19}+\eta_{20}\right] k^{T}\left(z\left(n-\tau_{1}\right)\right) \lambda_{\max }\left(B^{T} B\right) k\left(z\left(n-\tau_{1}\right)\right)\right. \\
& \left.+\left[1+\delta_{4}^{-1}+\delta_{9}^{-1}+\delta_{13}^{-1}+\delta_{16}+\delta_{19}+\delta_{20}\right] k^{T}\left(z\left(n-\tau_{1}\right)\right) \lambda_{\max }\left(A^{T} A\right) k\left(z\left(n-\tau_{1}\right)\right)\right\} \\
& +|p|^{2}\left\{\left[1+\eta_{5}^{-1}+\eta_{10}^{-1}+\eta_{14}^{-1}+\eta_{17}^{-1}+\eta_{19}^{-1}+\eta_{21}\right] h_{1}^{T}(z(n-\alpha)) \lambda_{\max }\left(A^{T} A\right) h_{1}(z(n-\alpha))\right. \\
& \left.+\left[1+\delta_{5}^{-1}+\delta_{10}^{-1}+\delta_{14}^{-1}+\delta_{17}^{-1}+\delta_{19}^{-1}+\delta_{21}\right] h_{1}^{T}(z(n-\alpha)) \lambda_{\max }\left(B^{T} B\right) h_{1}(z(n-\alpha))\right\} \\
& +|p|^{2}\left\{\left[1+\eta_{6}^{-1}+\eta_{11}^{-1}+\eta_{15}^{-1}+\eta_{18}^{-1}+\eta_{20}^{-1}+\eta_{21}^{-1}\right] k_{1}^{T}(z(n-\alpha)) \lambda_{\max }\left(B^{T} B\right) k_{1}(z(n-\alpha))\right. \text {. } \\
& \left.+\left[1+\delta_{6}^{-1}+\delta_{11}^{-1}+\delta_{15}^{-1}+\delta_{18}^{-1}+\delta_{20}^{-1}+\delta_{21}^{-1}\right] k_{1}^{T}(z(n-\alpha)) \lambda_{\max }\left(A^{T} A\right) k_{1}(z(n-\alpha)) .\right\}
\end{aligned}
$$

Using (16) we have

$$
\begin{aligned}
W(n+1) \leq & |\tilde{a}|^{2}\left\{\beta U^{T}(n) U(n)+\beta V^{T}(n) V(n)\right\} \\
& +k^{T}(z(n)) \beta\left(\lambda_{\max }\left(A^{T} A\right)+\lambda_{\max }\left(B^{T} B\right)\right) k(z(n)) \\
& +|c|^{2} \beta h^{T}\left(z\left(n-\tau_{1}\right)\right)\left(\lambda_{\max }\left(A^{T} A\right)+\lambda_{\max }\left(B^{T} B\right)\right) h\left(z\left(n-\tau_{1}\right)\right) \\
& +|c|^{2} \beta k^{T}\left(z\left(n-\tau_{1}\right)\right)\left(\lambda_{\max }\left(A^{T} A\right)+\lambda_{\max }\left(B^{T} B\right)\right) k\left(z\left(n-\tau_{1}\right)\right) \\
& +|p|^{2} \beta h_{1}^{T}(z(n-\alpha))\left(\lambda_{\max }\left(A^{T} A\right)+\lambda_{\max }\left(B^{T} B\right)\right) h_{1}(z(n-\alpha)) \\
& +|p|^{2} \beta k_{1}^{T}(z(n-\alpha))\left(\lambda_{\max }\left(A^{T} A\right)+\lambda_{\max }\left(B^{T} B\right)\right) k_{1}(z(n-\alpha))
\end{aligned}
$$

Further from the relations (20), and (18) we have

$$
\begin{aligned}
W(n+1) \leq & \beta|\tilde{a}|^{2} W(n)+2 \beta L^{2}\left\{\lambda_{\max }\left(A^{T} A\right)+\lambda_{\max }\left(B^{T} B\right)\right\} W(n) \\
& +2 \beta|c|^{2} L_{\max }^{2}\left\{\lambda_{\max }\left(B^{T} B\right)+\lambda_{\max }\left(A^{T} A\right)\right\}\left|\left(z\left(n-\tau_{1}\right)\right)\right|^{2} \\
& +2 \beta|p|^{2} L_{\max }^{2}\left\{\lambda_{\max }\left(B^{T} B\right)+\lambda_{\max }\left(A^{T} A\right)\right\}|(z(n-\alpha))|^{2} .
\end{aligned}
$$


Let $z(n-k)=\max \left\{z\left(n-\tau_{1}\right), z(n-\alpha)\right\}$.

$$
\begin{gathered}
W(n+1) \leq \beta|\tilde{a}|^{2} W(n)+2 \beta L^{2}\left\{\lambda_{\max }\left(A^{T} A\right)+\lambda_{\max }\left(B^{T} B\right)\right\} W(n) \\
+2 \beta\left[|c|^{2}+|p|^{2}\right] L_{\max }^{2}\left\{\lambda_{\max }\left(A^{T} A\right)+\lambda_{\max }\left(B^{T} B\right)\right\} \\
\max \{W(n), W(n-1), \ldots W(n-k)\} .
\end{gathered}
$$

Since

$$
\begin{aligned}
\Delta W(n)= & W(n+1)-W(n), \\
\leq & -\max _{1 \leq i \leq m}\left[1-\beta\left|\tilde{a}_{i}\right|^{2}-2 \beta\left|L_{i}\right|^{2}\left(\lambda_{\max }\left(A^{T} A\right)+\lambda_{\max }\left(B^{T} B\right)\right)\right] W(n) \\
& +2 \beta L_{\max }^{2}\left(\lambda_{\max }\left(c^{T} c\right)+\lambda_{\max }\left(p^{T} p\right)\right)\left[\left(\lambda_{\max }\left(A^{T} A\right)+\lambda_{\max }\left(B^{T} B\right)\right)\right] \\
& \times \max \{W(n), W(n-1), \ldots W(n-k)\} \\
\leq & -\tilde{p} W(n)+\tilde{q} \max \{W(n), W(n-1), \ldots W(n-k)\},
\end{aligned}
$$

where

$$
\begin{aligned}
& \tilde{p}=\max _{1 \leq i \leq m}\left[1-\beta\left|\tilde{a}_{i}\right|^{2}-2 \beta\left|L_{i}\right|^{2}\left(\lambda_{\max }\left(A^{T} A\right)+\lambda_{\max }\left(B^{T} B\right)\right)\right] \\
& \tilde{q}=4 \beta L_{\max }^{2}\left(\lambda_{\max }\left(c^{T} c\right)+\lambda_{\max }\left(p^{T} p\right)\right)\left[\left(\lambda_{\max }\left(A^{T} A\right)+\lambda_{\max }\left(B^{T} B\right)\right)\right] .
\end{aligned}
$$

From Lemma 3.1 , if $\tilde{q}<\tilde{p}$ there exist a constant $\lambda_{0} \in(0,1)$ such that

$$
\begin{aligned}
W(n) & =|z(n)|^{2}=z^{\mathrm{T}}(n) z(n)=\sum_{i=1}^{m}\left|z_{i}(n)\right|^{2} \\
& \leq \max \{W(0), W(-1), \ldots W(-k)\} \lambda_{0}^{n} \\
& \leq \max \left\{z_{i}(0), \sum_{i=1}^{m}\left|z_{i}(0)\right|^{2}, \sum_{i=1}^{m}\left|z_{i}(-1)\right|^{2}, \ldots \sum_{i=1}^{m}\left|z_{i}(-k)\right|^{2}\right\} \lambda_{0}^{n} \\
& \leq M\|\phi\|^{2} \lambda_{0}^{n},
\end{aligned}
$$

where $\|\phi\|^{2}=\max \left\{0, \sum_{i=1}^{m}\left|z_{i}(0)\right|^{2}, \sum_{i=1}^{m}\left|z_{i}(-1)\right|^{2}, \ldots \sum_{i=1}^{m}\left|z_{i}(-k)\right|^{2}\right\}$, and $M \geq 1$.

Hence the relation (24) establishes that the equilibrium point of (13) is globally exponentially stable.

Corollary 3.2: Assume that the conditions of Theorem 3.2 hold and $|\tilde{a}|<1$. Then (2) has a unique equilibrium $x^{*}$ and it is globally exponentially stable as solution of (2).

Proof: From (2) we have

$$
x(n+1)=\tilde{a} x(n)+\tilde{b} f(Z(n)) .
$$

Thus we have $x(n+1)-\tilde{a} x(n)=\tilde{b} f(Z(n))$. 
Using the method for solving non homogeneous difference equation, one can establish that

$$
x(n)=\tilde{a}^{n} x_{0}+\sum_{k=0}^{n-1} \tilde{a}^{n-k-1} \tilde{b} f(z(n)) .
$$

We let $n \rightarrow \infty$ to the equation (25) and obtain

$$
\lim _{n \rightarrow \infty} x(n)=\lim _{n \rightarrow \infty} \tilde{a}^{n} x_{0}+\lim _{n \rightarrow \infty} \sum_{k=0}^{n-1} \tilde{a}^{n-k-1} \tilde{b} f(z(n)) .
$$

Since $z(n) \rightarrow z^{*}$ as $n \rightarrow \infty$ and $\mathrm{f}$ is continuous we have $f(z(n)) \rightarrow f\left(z^{*}\right)$.

Thus $\lim _{n \rightarrow \infty} x(n)=\lim _{n \rightarrow \infty} a^{n} x_{0}+\sum_{n=0}^{\infty} \tilde{a}^{n} \tilde{b} f\left(z^{*}\right)$.

If $|\tilde{a}|<1$ then $\lim _{n \rightarrow \infty} x(n)=\frac{\tilde{b}}{1-\tilde{a}} f\left(z^{*}\right)$. We let $x^{*}=\lim _{n \rightarrow \infty} x(n)$. Then it follows from (3) that $\lim _{n \rightarrow \infty} p x\left(n-\sigma^{0}\right)-p x\left(n-\sigma^{1}\right)$ exists and equals to $z^{*}-(1+c) x^{*}-q$. From the boundedness of $x(n)$ one can establish that $\lim _{n \rightarrow \infty} p x\left(n-\sigma^{0}\right)-p x\left(n-\sigma^{1}\right)=0$. Therefore $z^{*}-(1+c) x^{*}+q$ and hence $x^{*}$ is equilibrium of $(2)$.

In the above analysis, we have obtained sufficient condition for the asymptotic stability of equilibrium pattern of discretised complex valued hysteretic neuron models. However that much of complexity may not be adequate for certain neuron models. In the next theorem we discuss about discrete-time real valued hysteretic neuron model.

From the system (13) we have

$$
\begin{aligned}
& z(n+1)=\tilde{a} z(n)+\operatorname{Mg}(z(n))+c \operatorname{Mg}\left(z\left(n-\tau_{1}\right)\right)+p \operatorname{Mg}(z(n-\alpha)) \\
& \text { where } \tilde{g}(z(n-\alpha))=g\left(z\left(n-\sigma^{0}\right)\right)-g\left(z\left(n-\sigma^{1}\right)\right)
\end{aligned}
$$

Clearly if $\mathrm{H} 1$ holds and from relation (14) we have

$$
\begin{aligned}
& |g(z(n))| \leq L|z(n)|, L=\left\{L_{i}\right\}_{1 \leq i \leq m} \text { and } \\
& |\tilde{g}(z(n-\alpha))| \leq L_{\max }|z(n-\alpha)| \text { and } L_{\max }=\max _{1 \leq i \leq m}\left\{L_{i}\right\} .
\end{aligned}
$$

Theorem 3.2: Assume that the condition $\mathrm{H} 1$ and (9) holds and that there exist positive numbers $\eta_{i}, i=1,2, \ldots, 6$. If

$$
\begin{gathered}
\tilde{q}=\beta L_{\max }^{2}\left(\lambda_{\max }\left(c^{\mathrm{T}} c\right)-\lambda_{\max }\left(p^{\mathrm{T}} p\right)\right) \lambda_{\max }\left(M^{\mathrm{T}} M\right) \\
<\tilde{p}=\max _{1 \leq i \leq m}\left\{1-\beta \tilde{a}_{1}^{2}-\beta \lambda_{\max }\left(M^{\mathrm{T}} M\right) L_{i}^{2}\right\},
\end{gathered}
$$

where $\lambda_{\max }(M)$ denotes the largest eigen value of a matrix $M$, 


$$
\beta=\max \left\{\begin{array}{l}
\left(1+\eta_{1}^{-1}+\eta_{2}^{-1}+\eta_{3}^{-1}\right),\left(1+\eta_{1}+\eta_{4}^{-1}+\eta_{5}^{-1}\right), \\
\left(1+\eta_{2}+\eta_{4}+\eta_{6}^{-1}\right),\left(1+\eta_{3}+\eta_{5}+\eta_{6}\right)
\end{array}\right\}
$$

and $L_{\max }=\max _{1 \leq i \leq m}\left\{L_{i}\right\}$. Then the equilibrium point of (26) is globally exponentially stable.

Proof: Consider the following function $W(n)=|Z(n)|^{2}$, so that

$$
\begin{aligned}
W(n+1)= & |Z(n+1)|^{2}=Z^{\mathrm{T}}(n+1) Z(n+1) . \\
= & \left(z(n)^{T} \tilde{a}^{T}+g(z(n))^{T} \mathrm{M}^{T}+g\left(z\left(n-\tau_{1}\right)^{T}\right) \mathrm{M}^{T} c^{T}+\tilde{g}(z(n-\alpha))^{T} \mathrm{M}^{T} p^{T}\right) \\
& \times \tilde{a} z(n)+\operatorname{Mg}(z(n))+c \mathrm{Mg}\left(z\left(n-\tau_{1}\right)\right)+p \operatorname{Mg}(z(n-\alpha))
\end{aligned}
$$

Simplifying and using the inequalities $x y+y x \leq \eta x^{2}+\eta^{-1} y^{2}$ for any vectors $x, y \in R^{m}$ and $\eta>0$, we get

$$
\begin{aligned}
W(n+1) \leq & \tilde{a}^{2}\left(1+\eta_{1}^{-1}+\eta_{2}^{-1}+\eta_{3}^{-1}\right)|z(n)|^{2} \\
& +\left(1+\eta_{1}+\eta_{4}^{-1}+\eta_{5}^{-1}\right) \lambda_{\max }\left(M^{T} M\right)|g(z(n))|^{2} \\
& +\left(1+\eta_{2}+\eta_{4}+\eta_{6}^{-1}\right) \lambda_{\max }\left(c^{T} c\right) \lambda_{\max }\left(M^{T} M\right)\left|g\left(z\left(n-\tau_{1}\right)\right)\right|^{2} \\
& +\left(1+\eta_{3}+\eta_{5}+\eta_{6}\right) \lambda_{\max }\left(p^{T} p\right) \lambda_{\max }\left(M^{T} M\right)|\tilde{g}(z(n-\alpha))|^{2} .
\end{aligned}
$$

From relations (26) to (28) we have

$$
\begin{aligned}
W(n+1) \leq & \beta|\tilde{a}|^{2}|z(n)|^{2}+\beta \lambda_{\max }\left(M^{T} M\right) L^{2}|z(n)|^{2} \\
& +\beta \lambda_{\max }\left(c^{T} c\right) \lambda_{\max }\left(M^{T} M\right) L_{\max }^{2}\left|z\left(n-\tau_{1}\right)\right|^{2} \\
& +\beta \lambda_{\max }\left(p^{T} p\right) \lambda_{\max }\left(M^{T} M\right) L_{\max }^{2}|z(n-\alpha)|^{2}
\end{aligned}
$$

Assuming $z(n-k)=\max \left\{z\left(n-\tau_{1}\right), z(n-\alpha)\right\}$ hence

$$
\begin{aligned}
W(n+1) \leq & \beta \tilde{a}^{2}|z(n)|^{2}+\beta \lambda_{\max }\left(M^{T} M\right) L^{2}|z(n)|^{2} \\
& +\beta\left(\lambda_{\max }\left(c^{T} c\right)+\lambda_{\max }\left(p^{T} p\right)\right) \lambda_{\max }\left(M^{T} M\right) L_{\max }^{2}|z(n-k)|^{2} .
\end{aligned}
$$

Thus

$$
\begin{aligned}
\Delta W(n)= & W(n+1)-W(n) \\
\leq & -\left\{I-\beta|\tilde{a}|^{2}-\beta \lambda_{\max }\left(M^{T} M\right) L^{2}\right\} W(n) \\
& +\beta L_{\max }^{2}\left(\lambda_{\max }\left(c^{T} c\right)+\lambda_{\max }\left(p^{T} p\right)\right) \lambda_{\max }\left(M^{T} M\right) \\
& \times \max \{W(n), W(n-1), \ldots W(n-k)\}
\end{aligned}
$$

where $I$ denotes an identity matrix with appropriate dimension. 
Simplifying we have

$$
\begin{aligned}
\Delta W(n) \leq & -\min \left\{I-\beta \tilde{a}_{i}^{2}-\beta \lambda_{\max }\left(M^{T} M\right) L_{i}^{2}\right\} W(n) \\
& +\beta L_{\max }^{2}\left(\lambda_{\max }\left(c^{T} c\right)+\lambda_{\max }\left(p^{T} p\right)\right) \lambda_{\max }\left(M^{T} M\right) \\
& \max \{W(n), W(n-1), \ldots W(n-k)\}
\end{aligned}
$$

So $\Delta W(n) \leq-\tilde{p} z(n)+\tilde{q} \max \{W(n), W(n-1), \ldots W(n-k)\}$, where

$$
\begin{aligned}
& \tilde{p}=\min \left\{I-\beta \tilde{a}_{i}^{2}-\beta \lambda_{\max }\left(M^{T} M\right) L_{i}^{2}\right\} \\
& \tilde{q}=\beta L_{\max }^{2}\left(\lambda_{\max }\left(c^{T} c\right)+\lambda_{\max }\left(p^{T} p\right)\right) \lambda_{\max }\left(M^{T} M\right) .
\end{aligned}
$$

From Lemma 3.1, if $\tilde{q}<\tilde{p}$ there exist a constant $\lambda_{0} \in(0,1)$ such that

$$
\begin{aligned}
W(n) & =|z(n)|^{2}=z^{\mathrm{T}}(n) z(n)=\sum_{i=1}^{m} z_{i}^{2}(n) \\
& \leq \max \{W(0), W(-1), \ldots W(-k)\} \lambda_{0}^{n} \\
& \leq \max \left\{z_{i}(0), \sum_{i=1}^{m} z_{i}^{2}(0), \sum_{i=1}^{m} z_{i}^{2}(-1), \ldots \sum_{i=1}^{m} z_{i}^{2}(-k)\right\} \lambda_{0}^{n} \\
& \leq M\|\phi\|^{2} \lambda_{0}^{n} .
\end{aligned}
$$

Hence the equilibrium point of (26) is globally exponentially stable.

Remark 3.1: Usually the delays in artificial neural networks are time varying due to the finite switching speed of amplifiers. So we can consider (1) with varying time delays. Assuming that the varying time delays $\sigma(t), \tau(t)$ are bounded and non negative functions. Therefore assuming the conditions of Theorem 3.1 hold and $\sigma(n), \tau(n)$ are bounded, following proof similar to Theorem 3.1 there exists global exponential stable equilibrium point for discrete-time of (1) with varying delays.

Example.3.1: Consider the following network with $\mathrm{z}(n)=u(n)+j v(n)$

$$
\begin{aligned}
Z(n+1)= & {\left[\begin{array}{cc}
0.25 & 0 \\
0 & 0.05
\end{array}\right] Z(n)+\left(\left[\begin{array}{cc}
0.025 & 0.01 \\
-0.004 & 0.025
\end{array}\right]+j\left[\begin{array}{cc}
0.005 & -0.002 \\
0.015 & -0.003
\end{array}\right]\right) g(z(n)) } \\
& +\left[\begin{array}{cc}
0.13 & 0 \\
0 & 0.25
\end{array}\right]\left(\left[\begin{array}{cc}
0.025 & 0.01 \\
-0.004 & -0.025
\end{array}\right]+j\left[\begin{array}{cc}
0.005 & -0.002 \\
0.015 & -0.003
\end{array}\right]\right) g\left(z\left(n-\tau_{1}\right)\right. \\
& +\left[\begin{array}{cc}
0.09 & 0 \\
0 & 0.03
\end{array}\right]\left(\left[\begin{array}{cc}
0.025 & 0.01 \\
-0.004 & -0.025
\end{array}\right]+j\left[\begin{array}{cc}
0.005 & -0.002 \\
0.015 & -0.003
\end{array}\right]\right) h(z(n-\alpha))
\end{aligned}
$$

where $h(z(n-\alpha))=\left[g\left(z\left(n-\sigma^{0}\right)\right)-g\left(z\left(n-\sigma^{1}\right)\right)\right]$ and $g(z(n))=\tanh (z(n))$.

Network in Example 3.1 satisfies the $0<a_{0}=\max _{1 \leq i \leq m} \tilde{a}_{i}=0.25<1$ and

$0<a_{0}+\max _{1 \leq i \leq m}\left|1+c_{i}\right| \sum_{k=1}^{m} L_{k}\left|\tilde{b}_{i k}\right|=0.25108125<1$. 
Activation function $g(z(n))$ satisfies the hypothesis of Theorem 3.1 with $L_{i}=1$ and $\eta_{i}=1, \delta_{i}=1, \underline{i}=1,2, \ldots 21$, so $\beta=7$ and

$$
\begin{aligned}
& \lambda_{\max }\left(A^{T} A\right)=0.001036, \lambda_{\max }\left(B^{T} B\right)=0.000262 \\
& \text { So } \lambda_{\max }\left(A^{T} A\right)+\lambda_{\max }\left(B^{T} B\right)=0.001298 \\
& \lambda_{\max }\left(c^{T} c\right)=0.000625, \lambda_{\max }\left(p^{T} p\right)=0.000081 \\
& \text { So } \lambda_{\max }\left(c^{T} c\right)+\lambda_{\max }\left(p^{T} p\right)=0.000706 \\
& \tilde{q}=4 \beta L_{\max }^{2}\left(\lambda_{\max }\left(c^{T} c\right)+\lambda_{\max }\left(p^{T} p\right)\right)\left[\lambda_{\max }\left(A^{T} A\right)+\lambda_{\max }\left(B^{T} B\right)\right] \\
& \quad=0.000024<0.5457 \\
& \quad=\min \left[1-\beta\left|\tilde{a}_{i}\right|^{2}-2 \beta\left|L_{i}\right|^{2}\left(\lambda_{\max }\left(A^{T} A\right)+\lambda_{\max }\left(B^{T} B\right)\right)\right] \\
& \quad=\tilde{p}
\end{aligned}
$$

Hence the network in Example 3.1 satisfies the conditions of Theorem 3.1.

Example.3.2: Consider the network

$$
\begin{aligned}
Z(n+1)= & {\left[\begin{array}{cc}
0.3 & 0 \\
0 & 0.1
\end{array}\right] Z(n)+\left[\begin{array}{cc}
0.125 & -0.1 \\
0.01 & -0.25
\end{array}\right] g(z(n)) } \\
& +\left[\begin{array}{cc}
0.3 & 0 \\
0 & 0.05
\end{array}\right]\left[\begin{array}{cc}
0.125 & -0.1 \\
0.01 & -0.25
\end{array}\right] g\left(z\left(n-\tau_{1}\right)\right. \\
& +\left[\begin{array}{cc}
0.01 & 0 \\
0 & 0.02
\end{array}\right]\left[\begin{array}{cc}
0.125 & -0.1 \\
0.01 & -0.25
\end{array}\right] h(z(n-\alpha)),
\end{aligned}
$$

where $h(z(n-\alpha))=\left[g\left(z\left(n-\sigma^{0}\right)\right)-g\left(z\left(n-\sigma^{1}\right)\right)\right]$ and $g(z(n))=\tanh (z(n))$.

Activation function $g(z(n))$ satisfies the hypothesis of Theorem 3.2 with $L_{i}=1$ and $\eta_{i}=1, \delta_{i}=1, \underline{i}=1,2, \ldots 6$, so $\beta=4$.

Network in Example 3.2 satisfies the $0<a_{0}=\max _{1 \leq i \leq m} \tilde{a}_{i}=0.3<1$ and $0<a_{0}+\max _{1 \leq i \leq m}\left|1+c_{i}\right| \sum_{k=1}^{m} L_{k}\left|\tilde{b}_{i k}\right|=0.573<1$.

Since

$$
\begin{aligned}
\lambda_{\max } & \left(M^{T} M\right)=0.0762, \lambda_{\max }\left(p^{T} p\right)=0.0004, \lambda_{\max }\left(c^{T} c\right)=0.0025 \\
\tilde{q} & =\beta L_{\max }^{2} \lambda_{\max }\left(p^{T} p\right) \lambda_{\max }\left(M^{T} M\right) \\
& =0.00088392<0.3286 \\
& =\max _{1 \leq i \leq m}\left\{1-\beta\left|\tilde{a}_{i}\right|^{2}+\beta \lambda_{\max }\left(M^{T} M\right) L_{i}^{2}\right\} \\
& =\tilde{p} .
\end{aligned}
$$


Hence the network in Example 3.2 satisfies the conditions of Theorem 3.2.

Figure 1 Solution of the network in Example 3.1

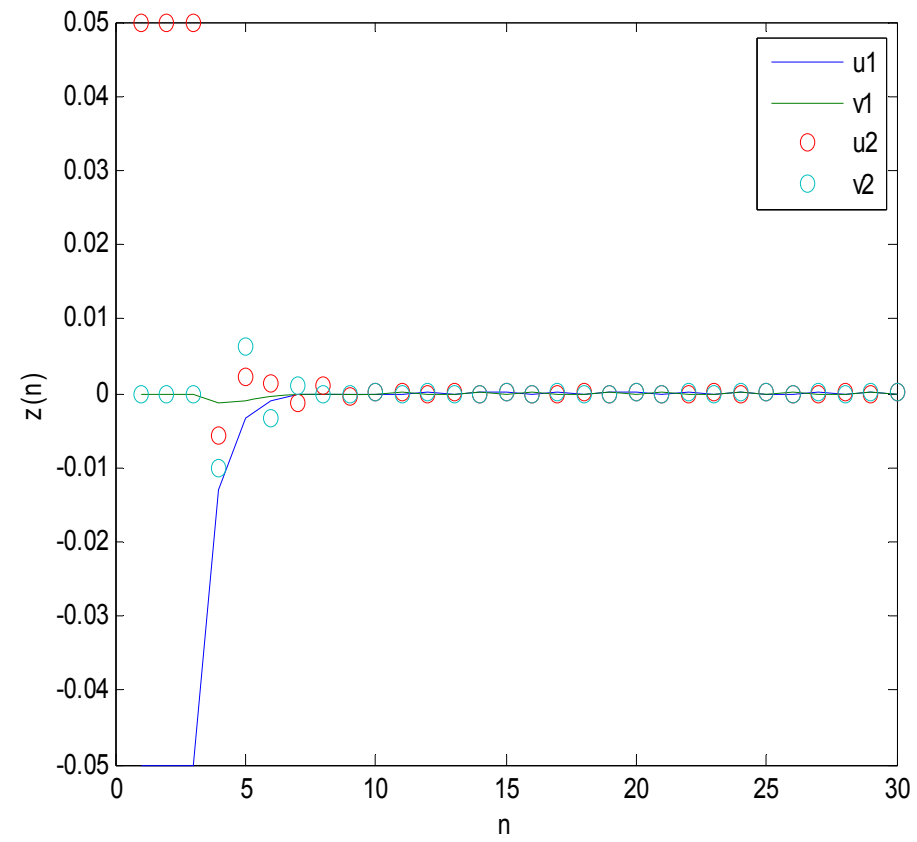

Figure 2 Solution of the network in Example 3.2

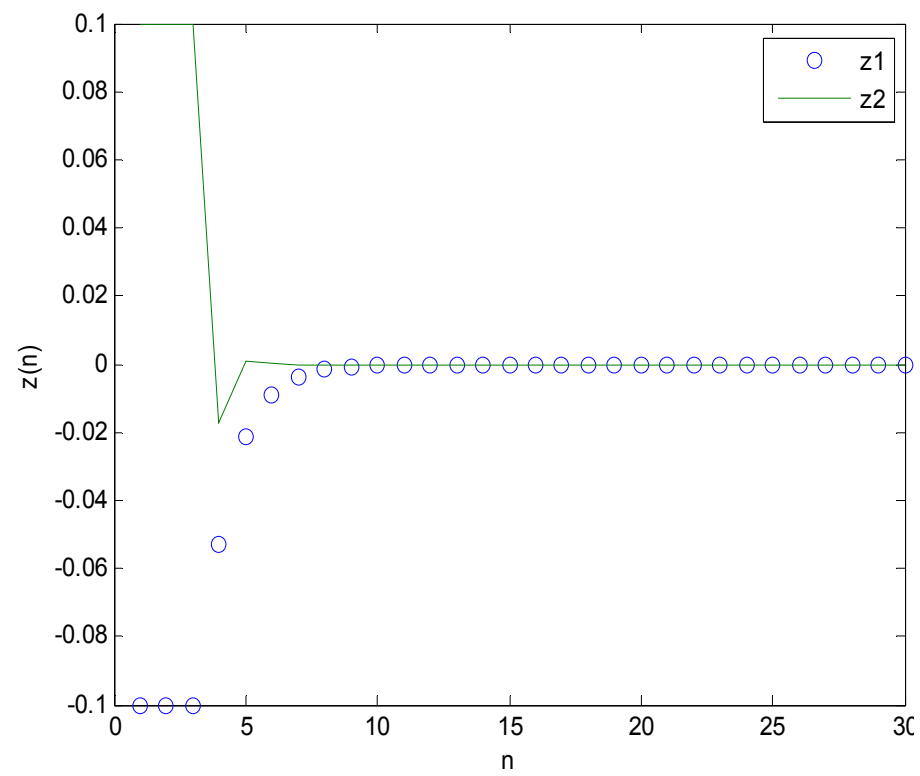




\section{Concluding remarks}

In this paper, we presented a discrete-time real and complex value hysteretic neuron model by applying Mohamad and Gopalsamy (Gopalsamy and Mohamad, 2003) discretisation technique on the continuous hysteretic neuron model. Global exponential stability is much desired for systems operating in real world constraints. We have obtained sufficient conditions for global exponential stability of a unique equilibrium and showed that even after discretisation the equilibrium of the system is stable. The results were demonstrated and verified with a set of practical numerical examples. The results are explicit in the sense that the criteria obtained are easily verifiable as they are expressed in terms of the parameters of the system. The hysteretic neural network model is envisaged to be efficient and robust for various applications such as medical, image processing, military data processing, etc. Hysteresis is a frequent phenomenon with implications for both everyday life as well as life sciences. Some situations like traffic jams occur when the car density exceeds a certain threshold value; return to free-flow traffic requires the car density to drop beyond a jam-triggering level. Our results can apply to verify the stability properties of the networks that arise both in the field of biological and artificial neural networks.

\section{Acknowledgements}

The authors would like to thank A.P. James, V. Sree Hari Rao, S. Raja Ratnam and two anonymous reviewers for the valuable comments they have given on an earlier version of this paper. The authors acknowledge the financial support of this work from CMSDSTGOI project Lr.No SR/S4/MS: 516/07 dated 21.04.2008.

\section{References}

Didday, R.L. (1976) 'A model of visuomotor mechanisms in the frog optic tectum', Math. Biosci., Vol. 30, Nos. 1-2, pp.169-180.

Feckan, M. (1999) 'Periodic solutions in systems at resonances with small relay hysteresis', Math. Slovaca, Vol. 49, No. 1, pp.41-52.

Georgion, M. and Koutsougeras, C. (1992) 'Complex domain back propagation', IEEE Trans. Circuits and Systems II: Analog and Digital Signal Processing, Vol. 39, No. 5, pp.330-334.

Gopalsamy, K. and Liu, P. (2007) 'Dynamics of hysteretic neuron', Nonlinear Analysis, Vol. 8, No. 1, pp.375-398.

Gopalsamy, K. and Mohamad, S. (2003) 'Exponential stability of continuous-time and discretetime cellular neural networks with delays', Appl. Math. Com., Vol. 135, No. 1, pp.17-38.

Hale, J. (1977) Theory of Functional Differential Equations, Springer-Verlag, New York.

Hirose, A. (2003) Complex-valued Neural Networks, Theories and Applications, World Scientific, Singapore.

Hoffman, G.W. (1986) 'A neural network model based on the analogy with the immune system', J. Theoret. Biol., Vol. 122, pp.33-67.

Hoffman, G.W. and Benson, H.W. (1986) 'Neurons with hysteresis from a network that can learn without any changes in synaptic strengths', in J.S. Denker (Ed.): Proc. Amer. Inst. Phys. Conf. Neural Network, Comput., pp.219-226. 
Krasnosel'skii, M.A. and Pokrovskii, A.V. (1989) Systems with Hysteresis, Springer-verlag, Berlin, New York.

Liz, E. and Ferreiro, J.B. (2002) 'A note on the global stability of generalized difference equations', Applied Mathematics Letters, Vol. 15, pp.655-659.

Macki, J.W., Nistri, P. and Zecca, P. (1993) 'Mathematical models for hysteresis', SIAM Review, Vol. 35, pp.94-123.

Nitta, T. (1997) 'An extension of the back-propagation algorithm to complex numbers', Neural Netw., Vol. 10, No. 8, pp.1391-1415.

Segundo, J.P. and Martinez, O.D. (1985) 'Dynamic and static hysteresis in crayfish receptors', Biol. Cybern., Vol. 52, No. 5, pp.291-296.

Sreeharirao, V. and Ramamurthy, G. (2008) 'Global dynamics of a class of complex valued neural networks', International Journal of Neural Systems, Vol. 18, No. 2, pp.165-171.

Zhang, Q., Wei, X. and Xu, J. (2006) 'A novel global exponential stability result for discrete-time cellular neural networks with variable delays', International Journal of Neural Systems, Vol. 16, No. 6, pp.467-472. 